\title{
Curva de Aprendizado ou de Aperfeiçoamento?
}

Ver artigo relacionado
na página 115

\author{
Roberto V. Botelho ${ }^{1}$, Clauber Lourenço ${ }^{1}$
}

D esde sua introdução para angiografia diagnóstica, por Campeau', em 1989, e para intervenções coronárias terapêuticas, por Kiemeneij e Laarman $^{2}$, em 1993, a via radial tem sido ampla e progressivamente utilizada em procedimentos endovasculares percutâneos, sejam cardíacos ou extracardíacos ${ }^{3}$. Vários e consistentes são os argumentos fomentadores dessa tendência universal, em comparação ao acesso braquial ou femoral, dentre eles estão a maior comodidade ao paciente, aferida pela maior qualidade de vida, percepção de desconforto, dor, ou mesmo, maior agilidade após o procedimento ${ }^{4}$. A possibilidade de deambulação precoce: nenhuma outra alternativa oferece deambulação tão rápida a tamanha parcela de pacientes; os dispositivos de sutura da artéria femoral não proporcionam tal precocidade à deambulação, tampouco o fazem à grande parcela dos candidatos ao método. Além disso, o acesso radial apresenta menores complicações, principalmente aquelas conseqüentes ao uso da terapia antitrombótica vigorosa, em síndromes coronárias agudas ${ }^{5}$. A vantagem da via radial é ainda mais legitimada ao se aferirem as complicações à luz da ultra-sonografia vascular, capaz de diagnosticar complicações não percebidas ao exame clínico desarmado. A relação custo/efetividade também é favorável ao acesso radial, haja vista a redução do tempo de internação hospitalar com esta via e dos custos derivados das complicações durante os acessos femorais ou braquiais ${ }^{6}$. Imprescindível aos laboratórios de cardiologia intervencionista é a ampliação de indicações, quando a via femoral é contra-indicada ou apresenta limitações ${ }^{5}$. Neste aspecto, até dez por cento dos pacientes não se candidatam ao acesso femoral, seja por doença aorto-ilíaca, anticoagulação sistêmica, obesidade mórbida, entre outros ${ }^{7}$. Nenhum intervencionista pode prescindir do domínio técnico dessa abordagem, tanto mais quando os avanços técnicos e tecnológicos criam instrumental cada vez mais apropriado, reduzindo eventuais limitações, como agulhas e jelcos específicos para a punção da artéria radial, guias metálicos com pontas mais macias e corpos de maior suporte, introdutores revestidos com polímeros lubrificantes, cateteres de menor calibre e com curvaturas multifuncionais (aptos a cateterizar seletivamente ambas as artérias coronárias, mesmo que com curvas pré-formadas), inúmeros dispositivos inteligentes para a compressão seletiva da artéria radial, poupando a artéria ulnar e mantendo o adequado fluxo do arco palmar, etc ${ }^{8}$. Por isso, essa via tem sido empregada em diferentes situações diagnósticas e terapêuticas, servindo ao tratamento de lesões complexas com aterectomias, abordagem de bifurcações, intervenções extracardíacas, em artérias carótidas, renais ou outros vasos maiores ${ }^{3}$. Em situações de emergência, como o infarto agudo do miocárdio, obtêm-se resultados semelhantes quanto aos índices de sucesso, porém, com significativa redução das complicações, principalmente aquelas relacionadas ao sítio da punção e ao uso de potente farmacologia antitrombínica e antiplaquetária ${ }^{9}$. Em nosso meio, avaliamos, em estudo randomizado e prospectivo, 600 pacientes submetidos à angioplastia primária, no infarto agudo do miocárdio, comparando a via radial à femoral. Os tempos porta-balão foram semelhantes, assim como os índices de sucesso e de fluxo TIMI $3^{10}$.

Entre as limitações, a ausência de boa suplência do arco palmar pela artéria ulnar pode restringir até $7 \%$ das indicações, quando se utiliza o teste de Allen manual. Essa limitação pode ser amenizada pelo teste oximétrico, quando menos que $2 \%$ dos pacientes se apresentam com o teste inadequado, portanto, negativo ${ }^{11}$.

A técnica é contra-indicada a pacientes com insuficiência renal, assim como àqueles com arterites ou fenômeno de Raynaud. A reutilização desta via é limitada, pois, mesmo que possível, à medida que seja reutilizada, a artéria radial sofre progressiva redução de calibre por remodelamento e espessamento intimal ${ }^{12}$, até a inviabilidade de nova punção, geralmente após a sétima utilização ${ }^{13}$.

A dose de radiação é, geralmente, maior que aquela oferecida pela via femoral, mesmo que o tempo de fluoroscopia seja semelhante, pois o operador trabalha mais próximo ao tubo de raios- $X^{14}$. A curva de aprendi-

\footnotetext{
${ }^{1}$ Instituto do Coração do Triângulo, Uberlândia, MG.

Correspondência: Dr. Roberto Botelho. Instituto do Coração do Triângulo. Rua Arthur Bernardes, 555 - Martins - Uberlândia, MG - CEP $38400-368$ Tel.: (34) 3239-8700 • E-mail: r.botelho@itms.com.br

Recebido em: 07/05/2007 - Aceito em: 08/05/2007
} 
zado é maior que a exigida pela via femoral e tem sido fonte de resistência de alguns operadores. Entretanto, deve-se atentar para inúmeras variáveis que interferem nessa análise e justificam a controvérsia e o debate sobre o real impacto desta curva de aprendizado. A principal causa de insucesso é a falha na canulação da artéria radial, responsável por até $80 \%$ dos insucessos. A simples sedação superficial dos pacientes, promovendo menor incidência de espasmos arteriais, pode interferir significativamente sobre essa variável. A utilização de jelcos de menor calibre também aumenta o sucesso do procedimento. Uma vez canulada a artéria, os espasmos podem ser reduzidos ao se empregarem introdutores lubrificados com silicone, assim como ao se utilizar um só cateter, evitando-se as trocas. O trabaIho de Nunes et al. ${ }^{15}$ apresenta interessante análise da redução de complicações ao longo do tempo, sugerindo curva de aprendizado de até 500 casos. Porém, em sua análise, englobam-se experiências de quatro operadores e não se discriminou o impacto do incremento técnico e tecnológico ao longo do tempo, o que pode reduzir esse período de aprendizado. A redução de insucessos e de complicações pode se dever a incrementos técnicos e tecnológicos, muito mais que à simples curva de aprendizado. Talvez represente curva de aperfeiçoamento, muito mais que de aprendizado, o que pode ocorrer com qualquer técnica, mesmo após seu amadurecimento. $O$ índice de sucesso dos primeiros 500 casos da experiência de Nunes et al. ${ }^{15}$ já é semelhante àquele apresentado pelo acesso braquial no estudo pioneiro Access ${ }^{16}$, em torno de 95\%. Da mesma forma, este índice, após os 4000 casos de Nunes et al. ${ }^{15}$, se aproxima dos melhores resultados do acesso femoral no mesmo estudo Access, em torno de $99 \%$.

Seja aprendizado ou aperfeiçoamento, a técnica atinge sua maioridade, ao se completarem 18 anos de sua introdução por Campeau', com seu lugar solidamente estabelecido nos programas de treinamento, assim como na prática diária dos diferentes centros de cardiologia intervencionista.

\section{REFERÊNCIAS BIBLIOGRÁFICAS}

1. Campeau L. Percutaneous radial artery approach for coronary angiography. Cathet Cardiovasc Diagn. 1989;16(1):3-7.

2. Kiemeneij F, Laarman G). Percutaneous transradial artery approach for coronary stent implantation. Cathet Cardiovasc Diagn. 1993;30(2):173-8.

3. Sharma GL, Louvard Y, Morice MC, Lefevre T, Loubeyre C, Dumas $\mathrm{P}$, et al. Noncoronary transradial angioplasty with coronary equipment: a less invasive technique. Catheter Cardiovasc Interv. 2002;55(2):197-205.
4. Cooper CJ, El-Shiekh RA, Cohen DJ, Blaesing L, Burket MW, Basu A, et al. Effect of transradial access on quality of life and cost of cardiac catheterization: a randomized comparison. Am Heart J. 1999;138(3 Pt 1):430-6.

5. Choussat R, Black A, Bossi I, Fajadet J, Marco J. Vascular complications and clinical outcome after coronary angioplasty with platelet $\mathrm{Ilb} / \mathrm{Ill}$ a receptor blockade. Comparison of transradial vs transfemoral arterial access. Eur Heart J. 2000;21(8): 662-7.

6. Mann T, Cubeddu G, Bowen J, Schneider JE, Arrowood M, Newman WN, et al. Stenting in acute coronary syndromes: a comparison of radial versus femoral access sites. J Am Coll Cardiol. 1998;32(3):572-6.

7. McNulty PH, Ettinger SM, Field JM, Gilchrist IC, Chambers CE, Gascho JA. Cardiac catheterization in morbidly obese patients. Catheter Cardiovasc Interv. 2002;56(2):174-7.

8. Botelho RV. Novos materiais para intervenção coronária por via radial. [citado Maio 01 2007]; Disponível em: http:// www.tsbtele.com.br/terumo/terumo_radial_roberto/index.html

9. Saito S, Tanaka S, Hiroe $Y$, Miyashita $Y$, Takahashi S, Tanaka K, et al. Comparative study on transradial approach vs. transfemoral approach in primary stent implantation for patients with acute myocardial infarction: results of the test for myocardial infarction by prospective unicenter randomization for access sites (TEMPURA) trial. Catheter Cardiovasc Interv. 2003;59(1):26-33.

10. Pereira AS, Botelho RV, Alessi SB, Lourenço C, Cuba G. Angioplastia primária: estudo randomizado comparando abordagem radial $x$ femoral. In: XXIX Congresso da Sociedade Brasileira de Hemodinâmica e Cardiologia Intervencionista; 2007 Brasília, DF. Anais. Revista Brasileira de Cardiologia Invasiva.

11. Benit E, Vranckx P, Jaspers L, Jackmaert R, Poelmans C, Coninx R. Frequency of a positive modified Allen's test in 1,000 consecutive patients undergoing cardiac catheterization. Cathet Cardiovasc Diagn. 1996;38(4):352-4.

12. Nagai S, Abe S, Sato T, Hozawa K, Yuki K, Hanashima K, et al. Ultrasonic assessment of vascular complications in coronary angiography and angioplasty after transradial approach. Am J Cardiol. 1999;83(2):180-6.

13. Sakai H, Ikeda S, Harada T, Yonashiro S, Ozumi K, Ohe H, et al. Limitations of successive transradial approach in the same arm: the Japanese experience. Catheter Cardiovasc Interv. 2001;54(2):204-8.

14. Sandborg M, Fransson SG, Pettersson H. Evaluation of patient-absorbed doses during coronary angiography and intervention by femoral and radial artery access. Eur Radiol. 2004;14(4):653-8.

15. Kiemeneij F, Laarman GJ, Odekerken D, Slagboom T, van der Wicken R. A randomized comparison of percutaneous transluminal coronary angioplasty by the radial, brachial and femoral approaches: the Access study. J Am Coll Cardiol. $1997 ; 29(6): 1269-75$.

16. Nunes G, Oliveira AT, Alves L, Alfonso T. Influência da curva de aprendizado no sucesso e na ocorrência de complicações associadas aos procedimentos pela via radial. Rev Bras Cardiol Invas. 2007;15(2):115-8. 